\title{
Linear Time-invariant Feedback Operator for Mobile Robot Trajectory Tracking
}

\author{
Suruz Miah $^{1}$, Wail Gueaieb ${ }^{1}$, Peter A. Farkas ${ }^{1}$, Salah Al-Sharhan ${ }^{2}$, and Davide Spinello ${ }^{1}$ \\ ${ }^{1}$ School of Electrical Engineering and Computer Science, University of Ottawa, Ottawa, Ontario, K1N6N5, Canada \\ ${ }^{2}$ Gulf University For Science and Technology, Hawally 32093 , Kuwait \\ e-Mails: \{Suruz.Miah,Wail.Gueaieb,Davide.Spinello\}@uOttawa.ca, peterfarkas@ieee.org, AlsharhanS@gust.edu.kw
}

\begin{abstract}
In this paper we propose a linear time-invariant (LTI) state feedback operator for nonholonomic mobile robots to track a pre-defined trajectory on a 2D planar region. Designing an on-line smooth state feedback control law is still among the major challenges for solving the trajectory tracking problem of nonholonomic systems including differential drive mobile robots. To address this problem, numerous nonlinear feedback control laws have been proposed to date. Most of these feedback control laws yield online solutions to the trajectory tracking problem with satisfactory tracking errors asymptotically. In most cases, they suffer from an overwhelming degree of computational complexity even to track a 2D trajectory using a simple unicyclelike nonholonomic system. The proposed control law offers an advantage of being smooth and LTI state-feedback in addition to its capability to address problems of partially observed systems. The shortcoming of the proposed control law is that the LTI feedback operator is computed off-line before the robot applies its control signal into the left and right wheels through their actuators. The theoretical results are supported by computer simulations followed by experiments with an e-puck mobile robot.
\end{abstract}

\section{INTRODUCTION}

Due to the escalating demand for collecting scientific data and for monitoring hazardous environments without human intervention, mobile robots are being extensively deployed in space (e.g., Curiosity (rover) ${ }^{1}$ ), on land (e.g., Google driverless $\mathrm{car}^{2}$ ), underground (e.g., Related video: Atlas Copco ST14 LHD ${ }^{3}$ ) [1], and in the ocean (e.g., surfing $\operatorname{robot}^{4}$ ) in recent years. The motion control in tracking a pre-defined trajectory or in stabilizing on a fixed configuration are often challenging tasks in these robotic applications/platforms. In particular, most wheeled mobile robots are underactuated and developing a smooth state feedback control law to perform these tasks is still among the major challenges as dictated by Brockett's theorem [2]. Here we provide an offline solution to the problem defined by Brockett's theorem for nonholonomic mobile robots.

Numerous nonlinear control laws have been proposed in the literature to address the trajectory tracking problem of mobile robots, see [3], [4], [5] for backstepping methods, [6], [7], [8] for sliding mode control, [9], [10], [11] for moving horizon $\mathcal{H}_{\infty}$ tracking control coupled with disturbance effect,

\footnotetext{
${ }^{1}$ http://en.wikipedia.org/wiki/Curiosity_(rover)

${ }^{2} \mathrm{http} / / /$ en.wikipedia.org/wiki/Google_driverless_car

${ }^{3} \mathrm{http} / / / \mathrm{www}$.youtube.com/watch?v=YgEUrkY80-U

${ }^{4} \mathrm{http} / / /$ news.stanford.edu/news/2012/august/surfing-robot-082312.html
}

[12] for transverse function approach, [13], [14] for formation control, and [15] for optimal motion planning. A vector-field orientation feedback control method for a differentially driven wheeled vehicle has been demonstrated in [16]. These control laws are quite powerful to solve the tracking problems of nonholonomic mobile robots but most of them either suffer from overwhelming degrees of computational complexity or require relatively complex feedback laws even to control a simple unicycle-like vehicles, see [17] and some references therein. The controller parameters (similar to the feedback gain parameters in our current work) are iteratively determined by adopting several tuning methodologies proposed by the authors in [18], [19], [20], [21]. Recently, linear time-varying feedback laws in cooperation with optimal control theory are extensively used by Miah and Gueaieb, see [22], [23], [24] for solving both trajectory tracking and regulation problems of wheeled mobile robots in a unified manner. Here we extend these ideas for the case when the feedback operator is linear in state and is time-invariant, which provides a partial solution to the tracking and stabilization problems of nonholonomic mobile robots as pointed out by the Brockett's conditions in [2].

The main differences between the current work and our previously relevant published articles (see [25], [26], [22], [23], [24]) are the following. In [25], [26], both time-varying and time-invariant feedback laws are established but they are tailored towards solving the tracking problems of semilinear dynamic systems only, which are not applicable for nonlinear affine systems (differential drive mobile robots, for example) as in the current work. This issue is then overcome in our recent work [22], [23], [24] by developing measurement feedback laws, where the feedback operator is time-varying as opposed to the proposed feedback operator which is timeinvariant.

The rest of the paper is outlined as follows. Section II illustrates the mathematical modeling of a differential drive mobile robot and formulates the tracking problem on a $2 \mathrm{D}$ planar region. The details on determining the optimal linear time-invariant feedback operator which constitute a statefeedback law is given section III. Section IV gives the thorough evaluation of the theoretical results with some computer simulations. Finally, conclusions are drawn in section V.

Notations: Throughout this paper, we will denote scalars by non-bold letters. Vectors and matrices will be denoted by small and capital bold letters, respectively. For positive integers $m$ 
and $n, \mathbb{R}^{n}$ and $\mathbb{R}^{n \times m}$ are the vector and matrix spaces of dimension $n$ and $n \times m$, respectively, taking values from real line $\mathbb{R} .(\cdot)^{T}$ will denote the transposition of the quantity $(\cdot)$. The scalar product of two vectors, $\mathbf{x}, \mathbf{y} \in \mathbb{R}^{n}$ are given by $(\mathbf{x} \cdot \mathbf{y}) \equiv \mathbf{x}^{T} \mathbf{y} \equiv \sum_{i=1}^{n} x_{i} y_{i}$, where $x_{i}$ and $y_{i}$ are the $i$ th component of vectors $\mathbf{x}$ and $\mathbf{y}$, respectively. $\mathbf{x} \odot \mathbf{y}$ denotes the standard outer product of two vectors i.e., $\mathbf{x} \odot \mathbf{y}=\mathbf{x y}^{T} . \operatorname{Tr}(\cdot)$ denotes the trace of matrix $(\cdot) . \mathbf{X} \otimes \mathbf{Y}$ is the Kronecker product of two matrices $\mathbf{X}, \mathbf{Y} \in \mathbb{R}^{m \times n}$, where $\mathbb{R}^{m^{2} \times n^{2}} \ni \mathbf{Z}=\mathbf{X} \otimes \mathbf{Y}$. $C\left(\mathcal{I}, \mathbb{R}^{n}\right)$ is the class of all continuous function on interval $\mathcal{I}$ taking values from $\mathbb{R}^{n}$.

\section{Mobile Robot Model and Problem Statement}

We consider a circular shaped mobile robot with $(x, y)$ and $\theta$ being its position and the heading angle with respect to a ground-fixed inertial reference frame $X-Y$, respectively, as shown in Fig. 1. Following [23], let $u_{L}$ and $u_{R}$, respectively, be the robot's left and right wheel angular velocities. The robot's position is the midpoint of the wheelbase of length $l$ connecting the two lateral wheels along their axis. Let us

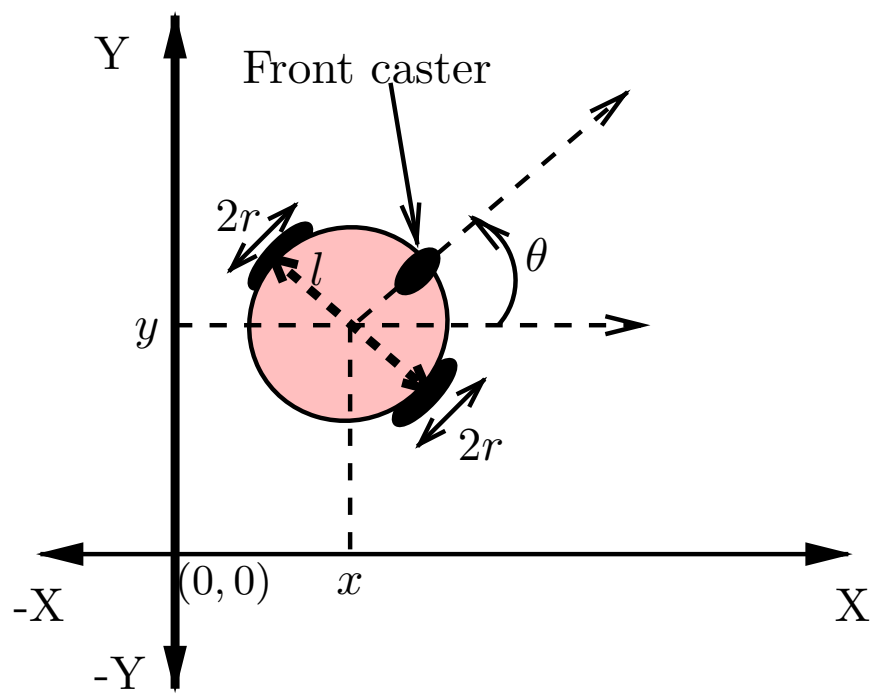

Fig. 1: Kinematic model of a differential-drive mobile robot.

denote $\mathcal{I}=\left[0, t_{f}\right]$ as the time interval with $0<t_{f}<\infty$ being the total time for the robot to track a pre-defined trajectory. At any time $t \in \mathcal{I}$, the robot kinematic model is given by

$$
\dot{\mathbf{q}}(t)=\mathbf{g}[\mathbf{q}(t), \mathbf{u}(t)]=\frac{r}{2} \mathbf{G}[\mathbf{q}(t)] \mathbf{u}(t),
$$

where $r$ is the radius of each wheel, the robot's configuration $\mathbf{q}(t) \equiv\left[\begin{array}{lll}x(t) & y(t) & \theta(t)\end{array}\right]^{T} \in \mathcal{Q} \subset \mathbb{R}^{2} \times \mathbb{S}^{1}$, its control input vector $\mathbf{u}(t) \equiv\left[u_{R}(t) u_{L}(t)\right]^{T} \in \mathcal{U} \subset \mathbb{R}^{2}, \theta(t) \in[-\pi,+\pi)$, and

$$
\mathbf{G}[\mathbf{q}(t)]=\left[\begin{array}{cc}
\cos \theta(t) & \cos \theta(t) \\
\sin \theta(t) & \sin \theta(t) \\
\frac{2}{l} & -\frac{2}{l}
\end{array}\right] .
$$

Due to the speed limits of the wheels, the inputs are constrained as

$$
\left|u_{L}(t)\right| \leq u_{L}^{\max } \text { and }\left|u_{R}(t)\right| \leq u_{R}^{\max }, t \in \mathcal{I} .
$$

In other words, $\mathbf{u}(t)$ must be chosen from a set of admissible speeds, $\mathcal{U}_{a d}$, i.e., $\mathbf{u}(t) \in \mathcal{U}_{a d}$. Note that a DDMR is a nonholonomic system with the nonholonomic constraint given by

$$
\dot{x} \sin \theta-\dot{y} \cos \theta=0,
$$

which ensures the wheel's non-slip movement in the lateral direction.

Let $\mathbf{q}^{d}(t)=\left[x^{d}(t) y^{d}(t) \theta^{d}(t)\right]^{T}$ be the desired trajectory and

$$
e(t)=\sqrt{\left[x^{d}(t)-x(t)\right]^{2}+\left[y^{d}(t)-y(t)\right]^{2}}
$$

denote its position tracking error, for $t \in \mathcal{I}$. The objective is to find the optimal control input $\mathbf{u}(t) \in \mathcal{U}_{a d}$ that generates the trajectory $\mathbf{q}(t) \in \mathcal{Q}$ while minimizing the total position tracking error, $\mathscr{E}$, given by

$$
\mathscr{E}=\int_{t_{0}}^{t_{f}} e(t) d t
$$

Given the robot's kinematic model (1), the speed constraint (2), and the nonholonomic constraint (3), the problem can be stated as follows:

$$
\inf _{\left\{\mathbf{q} \in \mathcal{Q}, \mathbf{u} \in \mathcal{U}_{a d}\right\}}[\mathscr{E}] .
$$

\section{Proposed LTI FeEdBack Operator Design}

This section presents the main results on determining the proposed linear time-invariant feedback operator for solving the trajectory tracking problem of a differential drive mobile robot. Assuming that the robot's full-state $\mathbf{q}(t)$ is available/measurable without noise at time $t \in \mathcal{I}$, let us define the full-state feedback law as

$$
\mathbf{u}(t)=\mathbf{K q}(t),
$$

where $\mathbf{K} \in \mathbb{R}^{2 \times 3}$ is the contant feedback operator that is to be determined for solving the problem (5). In the rest of the paper, we shall drop the argument $(t)$ for clarity when no ambiguity arises. Substituting the feedback law (6) in the robot's kinematic model (1), we get the robot's feedback model

$$
\dot{\mathbf{q}}=\mathbf{f}(\mathbf{q}, \mathbf{K})=\frac{r}{2} \mathbf{G}(\mathbf{q}) \mathbf{K q}, \quad \mathbf{q}(0)=\mathbf{q}_{0} .
$$

The actual trajectory of the feedback model (7) is described by

$$
\mathbf{q}(t)=\mathbf{q}_{0}+\int_{0}^{t_{f}} \mathbf{f}(\mathbf{q}, \mathbf{K}) \mathrm{dt} .
$$

For the robot's feedback model (7) to solve the problem (5), an appropriate value of the feedback operator $\mathbf{K}$ has to be available to the robot's controller. Note that there are two methods for determining the LTI feedback operator $\mathbf{K}$ :

1) The open-loop control through the classical Pontryagin's minimum principle (PMP) [27] and

2) The matrix-valued Pontryagin's minimum principle.

In the following, we illustrate these two methods for determining the optimal LTI feedback operator. 


\section{A. Determining K through Open-Loop Pontryagin's Mini- mum Principle}

The optimal feedback operator $\mathbf{K}$ can be determined by following the classical Pontryagin's minimum principle, where $\mathbf{K}$ is treated as the open-loop control input to the feedback system (7). For that, let $\operatorname{vec}(\mathbf{K})$ transforms the matrix $\mathbf{K}$ into a column vector, which is formed by stacking the rows of $\mathbf{K}$. The feedback model (7) can be rewritten as

$$
\dot{\mathbf{q}}=\mathbf{f}_{1}(\mathbf{q}, \overline{\mathbf{k}})=\frac{r}{2}\left(\mathbf{q}^{T} \otimes \mathbf{G}(\mathbf{q})\right) \overline{\mathbf{k}},
$$

where $\mathbb{R}^{6} \ni \overline{\mathbf{k}}=\operatorname{vec}(\mathbf{K})$ is now the control input vector. In order to solve the problem (5) using the PMP, we define the cost function as

$$
J(\overline{\mathbf{k}})=\phi\left(\mathbf{q}\left(t_{f}\right)\right)+\int_{0}^{t_{f}} \ell(\mathbf{q}, \overline{\mathbf{k}}) \mathrm{dt},
$$

where

$$
\begin{array}{r}
\phi\left(\mathbf{q}\left(t_{f}\right)\right)=\frac{1}{2}\left[\mathbf{q}\left(t_{f}\right)-\mathbf{q}^{d}\left(t_{f}\right)\right]^{T} \mathbf{P}\left(t_{f}\right)\left[\mathbf{q}\left(t_{f}\right)-\mathbf{q}^{d}\left(t_{f}\right)\right] \\
\ell(\mathbf{q}, \overline{\mathbf{k}})=\frac{1}{2}\left(\mathbf{q}-\mathbf{q}^{d}\right)^{T} \mathbf{Q}(t)\left(\mathbf{q}-\mathbf{q}^{d}\right)+\overline{\mathbf{k}}^{T} \mathbf{R}(t) \overline{\mathbf{k}}
\end{array}
$$

where $\mathbf{Q}$ and $\mathbf{R}$ are positive definite diagonal matrices that represent the relative importance of the robot's trajectory tracking performance and the actuator energy through the vector $\overline{\mathbf{k}}$. Note that the minimum $J$ corresponds to the minimum tracking error $\mathscr{E}$ defined in (4) depending on the appropriate choice of the matrices $\mathbf{Q}$ and $\mathbf{R}$. The matrix $\mathbf{P}\left(t_{f}\right)$ is also a positive definite matrix that signifies the relative importance of the strate tracking error at time $t_{f}$. In order to find the minimum $J$, we recast the feedback model (7) and the cost function (10) in terms of Hamiltonian defined by

$$
H(\mathbf{q}, \overline{\mathbf{k}}, \boldsymbol{\psi})=\boldsymbol{\psi}^{T} \mathbf{f}_{1}(\mathbf{q}, \overline{\mathbf{k}})+\ell(\mathbf{q}, \overline{\mathbf{k}}),
$$

where $\boldsymbol{\psi}(t) \equiv \boldsymbol{\psi} \in \mathbb{R}^{3}$ is the co-state vector that corresponds to the sensitivity of the cost function (10) due to state perturbations. Suppose that $\left(\mathbf{q}^{*}, \mathbf{k}^{*}\right)$ is the optimal trajectory and operator pair that minimizes the cost $J$. Following classical optimal control theory [28], [27], we can establish the following Pontryagin's minimum principle

$$
H\left(\mathbf{q}^{*}, \overline{\mathbf{k}}^{*}, \boldsymbol{\psi}^{*}\right) \leq H\left(\mathbf{q}^{*}, \overline{\mathbf{k}}, \boldsymbol{\psi}^{*}\right),
$$

$\forall \overline{\mathbf{k}} \in \mathbb{R}^{6}$. The inequality (11) can now be solved for the operator $\overline{\mathbf{k}}$ using the standard gradient descent technique presented in [28, Chapter 3] [27, Chaper 8]. By rearranging the elements of the optimal vector $\mathbf{k}^{*}$, we can reconstruct the optimal feedback operator matrix $\mathbf{K}^{*}$ which is then substituted in the feedback law (6) to generate the optimal control input vector $\mathbf{u}^{*}(t)$, for $t \in \mathcal{I}$. Note that the vector $\mathbf{u}^{*}(t)$ is then applied to the robot's actuator to generate optimal trajectory $\mathbf{q}^{*}(t)$ that minimizes the cost function (10), which eventually solves the problem (5).

\section{B. Determining K through Matrix-valued Pontryagin's Mini- mum Principle}

In the previous method, we determined the optimal LTI feedback operator $\mathbf{K}^{*}$ by first vectorizing the matrix $\mathbf{K}$, i.e., $\overline{\mathbf{k}}^{*}$, then the classical PMP is applied to determine $\mathbf{k}^{*}$. In this section, we determine the optimal feedback operator $\mathbf{K}^{*}$ directly by modifying the classical PMP. Inspired by the work on time-invariant feedback law for semi-linear dynamic systems [26], we introduce the cost functional as

$$
J(\mathbf{K})=\phi\left(\mathbf{q}\left(t_{f}\right)\right)+\int_{0}^{t_{f}} \ell(\mathbf{q}(t)) d t
$$

with

$$
\begin{aligned}
\phi\left(\mathbf{q}\left(t_{f}\right)\right) & =\frac{1}{2}\left[\mathbf{q}\left(t_{f}\right)-\mathbf{q}^{d}\left(t_{f}\right)\right]^{T} \mathbf{P}\left(t_{f}\right)\left[\mathbf{q}\left(t_{f}\right)-\mathbf{q}^{d}\left(t_{f}\right)\right] \\
\ell(\mathbf{q}(t)) & =\frac{1}{2}\left[\mathbf{q}(t)-\mathbf{q}^{d}(t)\right]^{T} \mathbf{Q}(t)\left[\mathbf{q}(t)-\mathbf{q}^{d}(t)\right],
\end{aligned}
$$

where $\mathbf{P}\left(t_{f}\right), \mathbf{Q}(t) \in \mathbb{R}^{3 \times 3}$ are symmetric positive definite matrices that indicate the relative importance of the error components along $\mathbb{R}^{2} \times \mathbb{S}^{1}$. If the robot's purpose is to reach a desired target point in its environment, then the weight matrix $\mathbf{P}\left(t_{f}\right)$ must be higher than $\mathbf{Q}(t)$. However, the opposte is true for the robot to track a desired trajectory. The performance index $J(\mathbf{K})$ in (12) depends on the feedback control operator matrix $\mathbf{K}$ through $\dot{\mathbf{q}}$, as it is clear from the feedback system (7). The problem is now to find the feedback operator $\mathbf{K}$ that generate the optimal trajectory $\mathbf{q}^{*}(t)$ while minimizing the cost function $J(\mathbf{K})$ in (12).

Assume that the feedback operator set $\mathcal{K} \subset \mathbb{R}^{2 \times 3}$ is closed bounded convex, and $\mathbf{K} \in \mathcal{K}$ such that the control input $\mathbf{u}(t)$ through (6) satisfies (2).

To solve for the optimal trajectory $\mathbf{q}^{*}(t), t \in \mathcal{I}$, using the feedback system (7), we need to derive the necessary conditions of optimality. These necessary conditions are most readily found if the integrand of the cost functional (12) is recast in terms of Hamiltonian $H: \mathbb{R}^{2} \times \mathbb{S}^{1} \times \mathbb{R}^{3} \times \mathbb{R}^{2 \times 3} \rightarrow \mathbb{R}$, which is expressed by

$$
H(\mathbf{q}, \boldsymbol{\psi}, \mathbf{K})=\boldsymbol{\psi}^{T} \mathbf{f}(\mathbf{q}, \mathbf{K})+\ell(\mathbf{q}),
$$

Similar to [29, Theorem 7.1, page 112], we derive the necessary conditions of optimality for the feedback model (7).

Theorem 1 (Necessary Conditions of Optimality): Given the feedback model (7) the trajectory $\mathbf{q}^{*}(t)$, the feedback operator $\mathbf{K}^{*} \in \mathcal{K}_{a d}$, and the co-state $\psi^{o}(t) \in C\left(\mathcal{I}, \mathbb{R}^{3}\right)$ are optimal with respect to $J(\mathbf{K})$ in (12) if:

$$
\begin{array}{r}
H\left(\mathbf{q}^{*}, \boldsymbol{\psi}^{*}, \mathbf{K}^{*}\right) \leq H\left(\mathbf{q}^{*}, \boldsymbol{\psi}^{*}, \mathbf{K}\right), \quad \forall \mathbf{K} \in \mathcal{K}, t \in \mathcal{I}, \\
\dot{\mathbf{q}}^{*}=\frac{\partial H}{\partial \boldsymbol{\psi}}\left(\mathbf{q}^{*}, \boldsymbol{\psi}^{*}, \mathbf{K}^{*}\right), \mathbf{q}^{*}(0)=\mathbf{q}_{0}, t \in \mathcal{I}, \\
\dot{\boldsymbol{\psi}}^{*}=-\frac{\partial H}{\partial \mathbf{q}}\left(\mathbf{q}^{*}, \boldsymbol{\psi}^{*}, \mathbf{K}^{*}\right), \boldsymbol{\psi}^{*}\left(t_{f}\right)=\frac{\partial \phi}{\partial \mathbf{q}}\left(\mathbf{q}\left(t_{f}\right)\right) .
\end{array}
$$

The proof is similar to the one presented in [29, Theorem 7.1,page 112]. Here we emphasize that the feedback 
operator $\mathbf{K}^{*}$ is linear in state $\mathbf{q}$ and is time-invariant. The inequality

$$
\frac{r}{2} \int_{0}^{t_{f}}\left(\boldsymbol{\psi}^{*}\right)^{T}\left(\mathbf{G}\left(\mathbf{K}-\mathbf{K}^{*}\right) \mathbf{q}\right) \mathrm{dt} \geq 0, \quad \forall \mathbf{K} \in \mathcal{K}_{a d}
$$

was derived in the proof of [29, Theorem 7.1,page 112], where the operator $\mathbf{K}$ was time-varying.

Theorem 1 states the necessary conditions for determining the optimal state trajectory $\mathbf{q}^{*}$ for the robot's feedback model (7) satisfying the Hamiltonian inequality (14) $\forall \mathbf{K} \in$ $\mathcal{K}_{a d}$. Note that the Hamiltonian inequality (14) is derived from the integral inequality (17). Hence, we establish the following Corollary to adaptively determine the optimal LTI feedback operator $\mathbf{K}^{*}$ that minimizes the cost functional (12).

Corollary 1 (Adapted from [26]): Consider the feedback system (7) defined over the time horizon $\mathcal{I}$. The following offline update rule for the feedback operator $\mathbf{K}$

$$
\begin{gathered}
\mathbf{K}_{\text {new }}=\mathbf{K}_{\text {old }}-\epsilon \mathbf{M}^{T}, \text { for } 0<\epsilon, \text { where } \\
\mathbf{M}=\int_{0}^{t_{f}}\left[\mathbf{q} \odot\left(\mathbf{G}^{T} \boldsymbol{\psi}^{*}\right)\right] \mathrm{dt} \in \mathbb{R}^{3 \times 2},
\end{gathered}
$$

satisfies the necessary condition (17) and, hence, guarantees the convergence of the robot's state towards its desired trajectory.

Proof: Inequality (17) can be rewritten as

$$
\int_{0}^{t_{f}}\left[\left(\left(\mathbf{K}-\mathbf{K}^{*}\right) \mathbf{q}^{*}\right) \cdot\left(\mathbf{G}^{T} \boldsymbol{\psi}^{*}\right)\right] \mathrm{dt} \geq 0 .
$$

Using the standard outer product the above inequality can be written as

$$
\int_{0}^{t_{f}} \operatorname{Tr}\left[\left(\mathbf{K}-\mathbf{K}^{*}\right)\left(\mathbf{q}^{*} \odot\left(\mathbf{G}^{T} \boldsymbol{\psi}^{*}\right)\right)\right] \mathrm{dt} \geq 0 .
$$

Inequality (20) can now be written as

$$
\begin{gathered}
\Upsilon(\mathbf{K}) \geq \Upsilon\left(\mathbf{K}^{*}\right), \text { where } \\
\Upsilon(\mathbf{K})=\operatorname{Tr}[\mathbf{K M}], \text { with } \mathbf{M}=\int_{0}^{t_{f}}\left[\mathbf{q} \odot\left(\mathbf{G}^{T} \boldsymbol{\psi}^{*}\right)\right] \mathrm{dt} .
\end{gathered}
$$

Using gradient descent method, the LTI feedback operator $\mathbf{K}$ can be iteratively determined as follows

$$
\mathbf{K}_{i+1}=\mathbf{K}_{i}-\epsilon \frac{\partial \Upsilon(\mathbf{K})}{\partial \mathbf{K}}=\mathbf{K}_{i}-\epsilon \mathbf{M}^{T}, \text { for } 0<\epsilon,
$$

which is the update rule (18), with $i$ being the offline iteration index, $\mathbf{K}_{i+1} \equiv \mathbf{K}_{n e w}$, and $\mathbf{K}_{i} \equiv \mathbf{K}_{\text {old }}$.

Using the above Corollary, we establish the key steps for the computation of the feedback operator $\mathbf{K}$ as follows.

\section{Numerical Computation of Feedback Operator $\mathbf{K}$}

Let $\mathbf{K}_{i} \in \mathcal{K} \subset \mathbb{R}^{2 \times 3}$, be the LTI feedback matrix at the $i$-th iteration. In the following, we optimize $\mathbf{K}_{i}$ using the gradient descent technique [28].

Step 0: Subdivide the time interval $\mathcal{I} \equiv\left[0, t_{f}\right]$ into $N$ equal subintervals.

Step 1: Integrate the feedback system (7) as in (8) using the initial condition $\mathbf{q}_{0}$ and the assumed $\mathbf{K}_{i}$; and record the solution as trajectory $\mathbf{q}_{i}(t) \equiv \mathbf{q}_{i}, t \in \mathcal{I}$.

Step 2: Integrate the costate equation (16) backward in time for $\boldsymbol{\psi}_{i} \forall t \in \mathcal{I}$.

Step 3: Define the Hamiltonian $H\left(\mathbf{q}_{i}, \boldsymbol{\psi}_{i}, \mathbf{K}_{i}\right)$ as in (13).

Step 4: Compute the cost functional $J\left(\mathbf{K}_{i}\right)$ using (12). Also compute the gradient $\partial \Upsilon / \partial \mathbf{K}=\mathbf{M}^{T}$.

Step 5: If $J\left(\mathbf{K}_{i}\right) \leq \delta$, then $\mathbf{K}_{i}$ is close to the optimal LTI feedback operator. Here $\delta$ is the pre-defined small positive number which is used as the tolerance (acceptable level of approximation) for the cost $J$.

Step 6: If $J\left(\mathbf{K}_{i}\right) \nless \delta \delta$, then use the following update rules to adjust the feedback control gain (or operator) $K_{i}$ :

$$
\begin{aligned}
\Delta \mathbf{K}_{i+1} & =\epsilon \frac{\partial \Upsilon(\mathbf{K})}{\partial \mathbf{K}}=\epsilon \Delta \mathbf{K}_{i} \text { and } \\
\mathbf{K}_{i+1} & =\mathbf{K}_{i}\left(t_{k}\right)-\Delta \mathbf{K}_{i+1},
\end{aligned}
$$

where $\epsilon$ is the step size, respectively. Replace $\mathbf{K}_{i}$ by $\mathbf{K}_{i+1}$ and return to Step 1.

In following section, we give computer simulations to implement above steps for a differential drive mobile robot to track a pre-defined desired trajectory.

\section{RESULTS}

The performance of the proposed linear time-invariant feedback operator $\mathbf{K}$ is evaluated by computer simulations followed by experiments with an e-puck robot shown in Figure $2^{5}$. The wheel base of the e-puck robot is $l=5.2 \mathrm{~cm}$ and the radius of its wheel is $r=2.05 \mathrm{~cm}$. The robot's wheel speeds are constrained as $u_{R}^{\max }=u_{L}^{\max }=7 \mathrm{rad} \cdot \mathrm{s}^{-1}$. For simplicity, we choose a straight line desired trajectory defined by $x^{d}(t)=(1 / 30) t-1$ and $y^{d}(t)=x^{d}+1$ that the robot is supposed to follow in $60 \mathrm{~s}$. The performance metric adopted in the current work is the cost defined in (12) which takes into account the tracking error between the robot's desired and actual trajectories over the time period of $I \equiv[0,60] \mathrm{s}$. Initially, the feedback control gain $\boldsymbol{K}$ is chosen as:

$$
\boldsymbol{K}=10^{-6}\left[\begin{array}{llll}
1 & 1 & 1 & 1 \\
1 & 1 & 1 & 1
\end{array}\right], \quad t \in[0,60] \mathrm{s} .
$$

The sampling time period is set to $0.4 \mathrm{~s}$. The weighting matrices of the cost function (12) are chosen as $\mathbf{P}\left(t_{f}\right)=$ $\operatorname{diag}(2,2,80)$ and $\mathbf{Q}(t)=\operatorname{diag}(40,40,60), \forall t \in[0,60] \mathrm{s}$. Hence, the trajectory tracking problem of the robot is given higher importance than just reaching the final destination. The feedback operator is then optimized offline using the steps

\footnotetext{
${ }^{5}$ Image of the e-puck robot is taken from http://en.wikipedia.org/wiki/Epuck_mobile_robot
} 


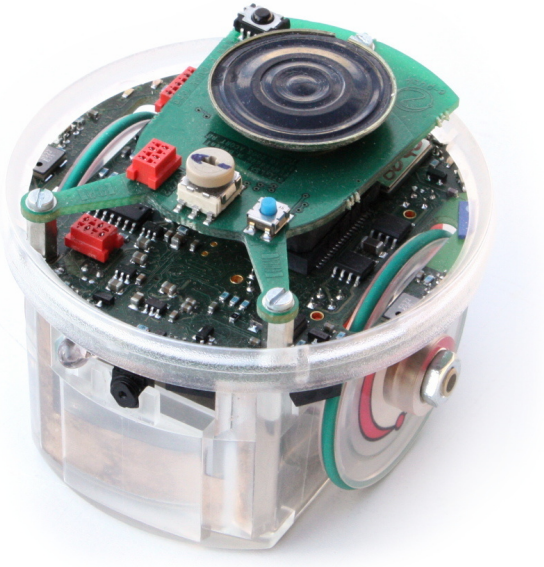

Fig. 2: E-puck robot used for experiments ${ }^{5}$.

discussed in section III-C. Initially, the robot is placed at position $(x, y)=(-1.5,0) \mathrm{m}$ with an orientation of $0^{\circ}$. The simulation results are demonstrated in Fig. 3. Despite the large initial cost (see Fig. 3(b)), the robot was able to converge to the target trajectory with an optimization cost of $J(\mathbf{K})=4.5$, which is practically nil compared to the initial cost. The actual and desire pose (position and orientation) of the robot is shown in Fig 3(a), where the dotted and solid lines represents the robot's actual and desired pose coordinates respectively. Since the robot's initial pose is perturbed from the desired initial pose, it took some time to converge to the desired trajectory (the initial desired and actual $y$-coordinates are same). It is clear that robot's speeds are within their speed limits, i.e., $u_{R}, u_{L} \leq 7 \mathrm{rad} \cdot \mathrm{s}^{-1}$. The computer simulation results shown in Fig. 3 are backed by real-time experiments with an e-puck robot summarized in Fig. 4. Fig. 4(a) shows the initial position of the robot which is $1.5 \mathrm{~m}$ off from the desired initial position. At time $t=30 \mathrm{~s}$, the robot is converging to the desired trajectory. It is worth noting that the robot is about $10 \mathrm{~cm}$ off from the final destination point $(1,2) \mathrm{m}$. This is desired according to the cost function (12) where $\mathbf{Q}$ is chosen to be higher than the $\mathbf{P}$ matrix. One has to compromise the cost of these two matrices depending on where the trajectory tracking problem is given higher priority than regulating to a fixed point or vice versa.

A silent feature of the proposed LTI feedback operator is that it can make to track any trajectory regardless of its complexity. This is, however, possible at the cost of choosing appropriate weighting parameters of the cost function (12).

\section{ACKNOWLEDGMENT}

The authors would like to acknowledge Dr. N. U. Ahmed for making us aware of this problem.

\section{CONCLUSION}

In this paper, we proposed a smooth linear time invariant feedback control law for a differential drive mobile robot to track a pre-defined trajectory on a planar region based on the
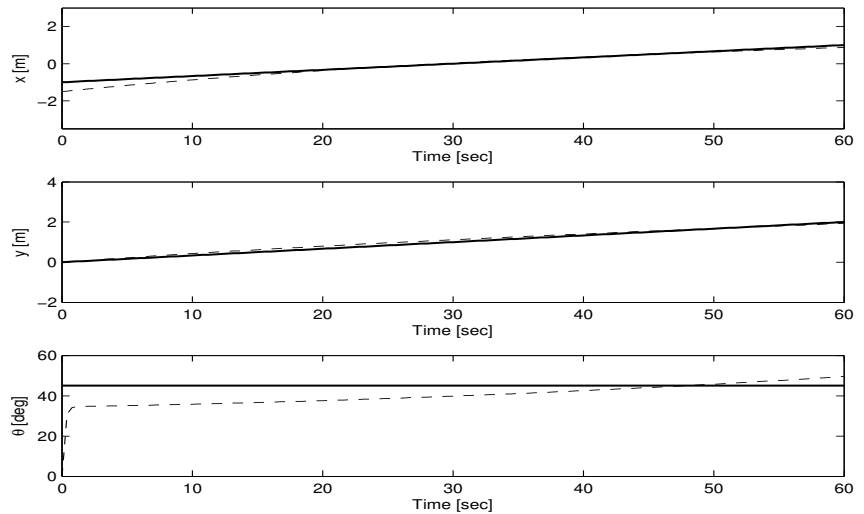

(a)

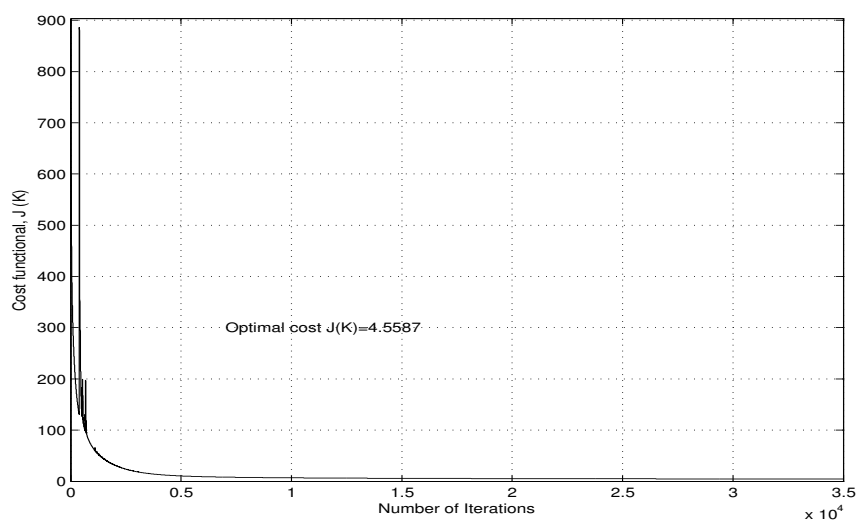

(b)

Fig. 3: Controller's performance in following a linear trajectory: (a) robot's pose trajectory $(x(t), y(t), \theta(t))$ (dotted line represents actual trajectory and solid line represents desired trajectory) and (b) cost, $J(\mathbf{K})$.

assumption that the robot's full-state is completely measurable without noise. One important observation that can be made from this feedback law is that it can be applied for solving both stabilization and tracking problems of nonlinear affine systems yielding offline solution to the Brockett's condition. Under certain conditions, it can be shown that the proposed LTI feedback law is able to stabilize a mobile robot on a fixed configuration regardless of its initial perturbation. The feedback law is also applicable for the case when the robot receives partial measurements about its state. The online implementation and the experimental validation of the proposed feedback control law are for future investigations.

\section{REFERENCES}

[1] J. Marshall, T. Barfoot, and J. Larsson, "Autonomous underground tramming for center-articulated vehicles," Journal of Field Robotics, vol. 25, no. 6-7, pp. 400-421, June-July 2008.

[2] R. W. Brockett, "Asymptotic stability and feedback stabilization," Differential Geometric Control Theory, pp. 181-191, 1983.

[3] D. Nganga-Kouya and F. Okou, "Adaptive backstepping control of a wheeled mobile robot," in 17th Mediterranean Conference on Control and Automation, Piscataway, NJ, USA, 2009, pp. 85-91.

[4] D. Chwa, "Tracking control of differential-drive wheeled mobile robots using a backstepping-like feedback linearization," IEEE Transactions on Systems, Man and Cybernetics, Part A: Systems and Humans, vol. 40, no. 6 , pp. 1285-1295, Nov. 2010. 


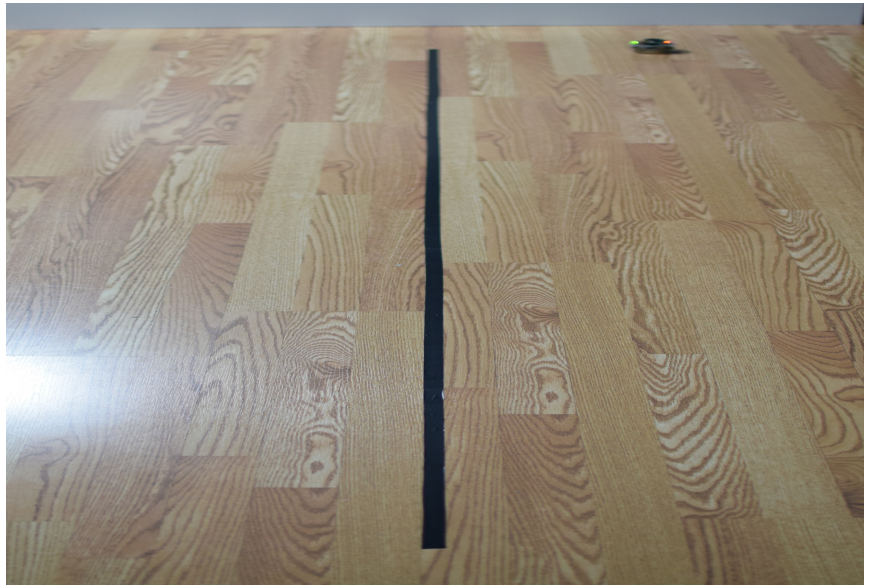

(a)

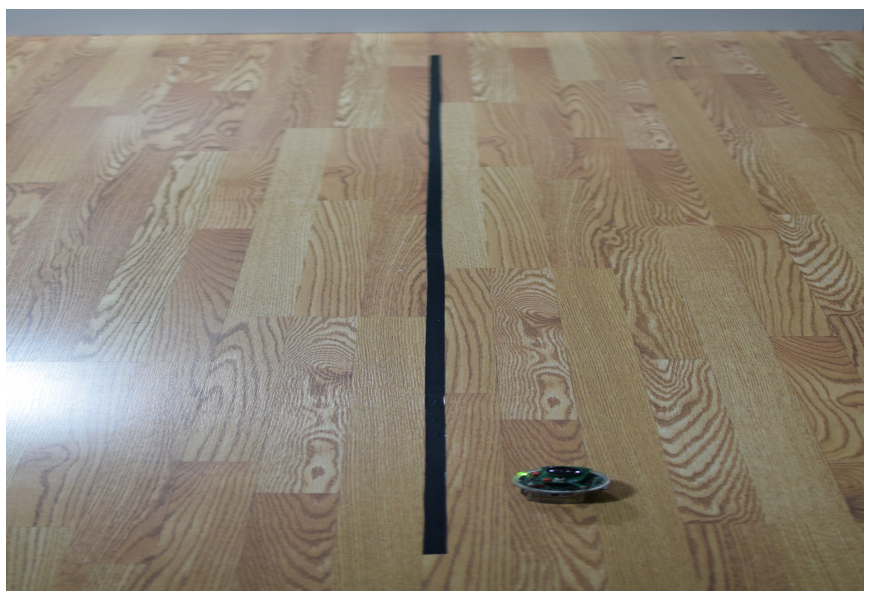

(b)

Fig. 4: Experimental results showing the controller's performance in following a linear trajectory: (a) robot's initial pose, (b) robot's pose at time $t=60 \mathrm{~s}$.

[5] Q. Cui, X. Li, X. Wang, and M. Zhang, "Backstepping control design on the dynamics of the omni-directional mobile robot," Applied Mechanics and Materials, vol. 203, pp. 51-6, 2012.

[6] J. H. Lee, C. Lin, H. Lim, and J. M. Lee, "Sliding mode control for trajectory tracking of mobile robot in the RFID sensor space," International Journal of Control, Automation and System, vol. 7, no. 3, pp. 429-435, Jun. 2009.

[7] M. Rubagotti, M. Della Vedova, and A. Ferrara, "Time-optimal slidingmode control of a mobile robot in a dynamic environment," IET Control Theory \& Applications, vol. 5, no. 16, pp. 1916-24, 2011.

[8] J.-X. Xu, Z.-Q. Guo, and T. H. Lee, "Design and implementation of integral sliding-mode control on an underactuated two-wheeled mobile robot," IEEE Transactions on Industrial Electronics, vol. 61, no. 7, pp. 3671-3681, July 2014.

[9] H. Chen, M.-M. Ma, H. Wang, Z.-Y. Liu, and Z.-X. Cai, "Moving horizon $\mathcal{H}_{\infty}$ tracking control of wheeled mobile robots with actuator saturation," IEEE Transactions on Control Systems Technology, vol. 17, no. 2, pp. 449-57, Mar. 2009.

[10] B. Kim, D. Necsulescu, and J. Sastadek, "Autonomous mobile robot model predictive control," International Journal of Control, vol. 77, no. 16, pp. 1438-45, 2004.

[11] R. Gonzalez, M. Fiacchini, T. Alamo, J. Guzman, and F. Rodriguez, "Online robust tube-based mpc for time-varying systems: a practical approach," International Journal of Control, vol. 84, no. 6, pp. 115770, 2011.

[12] P. Morin and C. Samson, "Control of nonholonomic mobile robots based on the transverse function approach," IEEE Transactions on Robotics, vol. 25 , no. 5 , pp. $1058-73$, Oct. 2009.

[13] J. Marshall, M. Broucke, and B. Francis, "Formations of vehicles in cyclic pursuit," IEEE Transactions on Automatic Control, vol. 49, no. 11, pp. 1963-1974, Nov 2004.

[14] K. Do, "Formation tracking control of unicycle-type mobile robots with limited sensing ranges," IEEE Transactions on Control Systems Technology, vol. 16, no. 3, pp. 527-538, May 2008.

[15] S. Liu and D. Sun, "Minimizing energy consumption of wheeled mobile robots via optimal motion planning," IEEE/ASME Transactions on Mechatronics, vol. 19, no. 2, pp. 401-411, April 2014.

[16] M. Michalek and K. Kozowski, "Vector-field-orientation feedback control method for a differentially driven vehicle," IEEE Transactions on Control Systems Technology, vol. 18, no. 1, pp. 45-65, jan 2010.

[17] A. Ailon and I. Zohar, "Control strategies for driving a group of nonholonomic kinematic mobile robots in formation along a time-parameterized path," IEEE/ASME Transactions on Mechatronics, vol. 17, no. 2, pp. 326-336, April 2012.

[18] J. C. Spall, "Multivariate stochastic approximation using a simultaneous perturbation gradient approximation," IEEE Transactions on Automatic Control, vol. 37, no. 3, pp. 332-341, 1992.

[19] S. Preitl, R.-E. Precup, J. Fodor, and B. Bede, "Iterative feedback tuning in fuzzy control systems. theory and applications," Acta Polytechnica Hungarica, vol. 3, no. 3, pp. 81-96, 2006.

[20] J. K. Huusom, N. K. Poulsen, and S. B. Jorgensen, "Iterative feedback tuning of uncertain state space systems," Brazilian Journal of Chemical Engineering, vol. 27, no. 3, pp. 461-472, 2010.

[21] Z. Hou and S. Jin, "Data-driven model-free adaptive control for a class of mimo nonlinear discrete-time systems," IEEE Transactions on Neural Networks, vol. 22, no. 12, pp. 2173-2188, 2011.

[22] M. S. Miah and W. Gueaieb, "Mobile robot trajectory tracking using noisy rss measurements: An rfid approach," ISA Transactions: The Journal of Automation, Elsevier, vol. 53, no. 2, pp. 433-443, mar 2014.

[23] M. Miah and W. Gueaieb, "RFID-based mobile robot trajectory tracking and point stabilization through on-line neighboring optimal control," Journal of Intelligent \& Robotic Systems, pp. 1-23, 2014. [Online]. Available: http://dx.doi.org/10.1007/s10846-014-0048-3

[24] M. S. Miah and W. Gueaieb, "Optimal time-varying p-controller for a class of uncertain nonlinear systems," International Journal of Control, Automation and Systems, vol. 12, no. 4, pp. 722-732, aug 2014.

[25] N. U. Ahmed and M. S. Miah, "Optimal feedback control law for a class of partially observed uncertain dynamic systems: A min-max problem," Dynamic Systems and Applications, vol. 20, no. 1, pp. 149-167, 2011.

[26] M. S. Miah and N. U. Ahmed, "Constant gain optimal output feedback control for a class of semi-linear dynamic systems," Dynamics of Continuous, Discrete and Impulsive Systems, Series B: Applications and Algorithms, vol. 19, no. 1, pp. 311-323, 2012.

[27] N. U. Ahmed, Dynamic Systems and Control with Applications. New Jersey: World Scientific, 2006.

[28] R. F. Stengel, Optimal Control and Estimation. Dover publications, inc., 1994.

[29] M. S. Miah, "Design and implementation of control techniques for differential drive mobile robots: An rfid approach," $\mathrm{PhD}$ dissertation, School of Electrical Engineering and Computer Science, University of Ottawa, 800 King Edward Avenue, Ottawa, Ontario, K1N 6N5, Canada, October 2012 